\title{
RADIATION DAMAGE TO BSCCO-2223 FROM 50 MEV PROTONS
}

\author{
A. F. Zeller ${ }^{1}$, R. M. Ronningen ${ }^{1}$, A. Godeke ${ }^{2}$, L. H. Heilbronn ${ }^{2}$, P. \\ McMahan-Norris $^{2}$, and R. Gupta ${ }^{3}$ \\ ${ }^{1}$ National Superconducting Cyclotron Lab \\ Michigan State University, E. Lansing, MI 48824 USA \\ ${ }^{2}$ Lawrence Berkeley National Lab \\ 1 Cyclotron Rd., Berkeley, CA 94720 USA \\ ${ }^{3}$ Brookhaven National Lab \\ Upton, NY 11973 USA
}

\begin{abstract}
The use of HTS materials in high radiation environments requires that the superconducting properties remain constant up to a radiation high dose. BSCCO-2223 samples from two manufacturers were irradiated with $50 \mathrm{MeV}$ protons at fluences of up to $5 \times 10^{17}$ protons $/ \mathrm{cm}^{2}$. The samples lost approximately $75 \%$ of their pre-irradiation $\mathrm{I}_{\mathrm{c}}$. This compares with $\mathrm{Nb}_{3} \mathrm{Sn}$, which loses about $50 \%$ at the same displacements per atom.
\end{abstract}

KEYWORDS: BSCCO-2223. Radiation damage.

\section{INTRODUCTION}

The magnets in the vicinity of the production target in a heavy ion fragment separator are subjected to very high doses of neutrons and other nuclear radiation. To maximize efficiency in capturing the rare isotopes coming from the production target the separator quadrupoles need high gradients and large apertures. These requirements necessitate using superconducting magnets. This leads to two problems: The short-term one is removing the nuclear heating so the magnets stay superconducting. The long-term one is having all components be resistant to damage. One solution [1] is to use a High Temperature Superconductor (HTS) in a superferric quadrupole. This addresses both problems because the coil can operate at $20-30 \mathrm{~K}$, with higher thermodynamic efficiency, and the electrical insulation can be stainless steel. Normally, the insulation is the most radiation sensitive of the materials used in a superconducting coil, but the use of stainless steel alleviates this. 


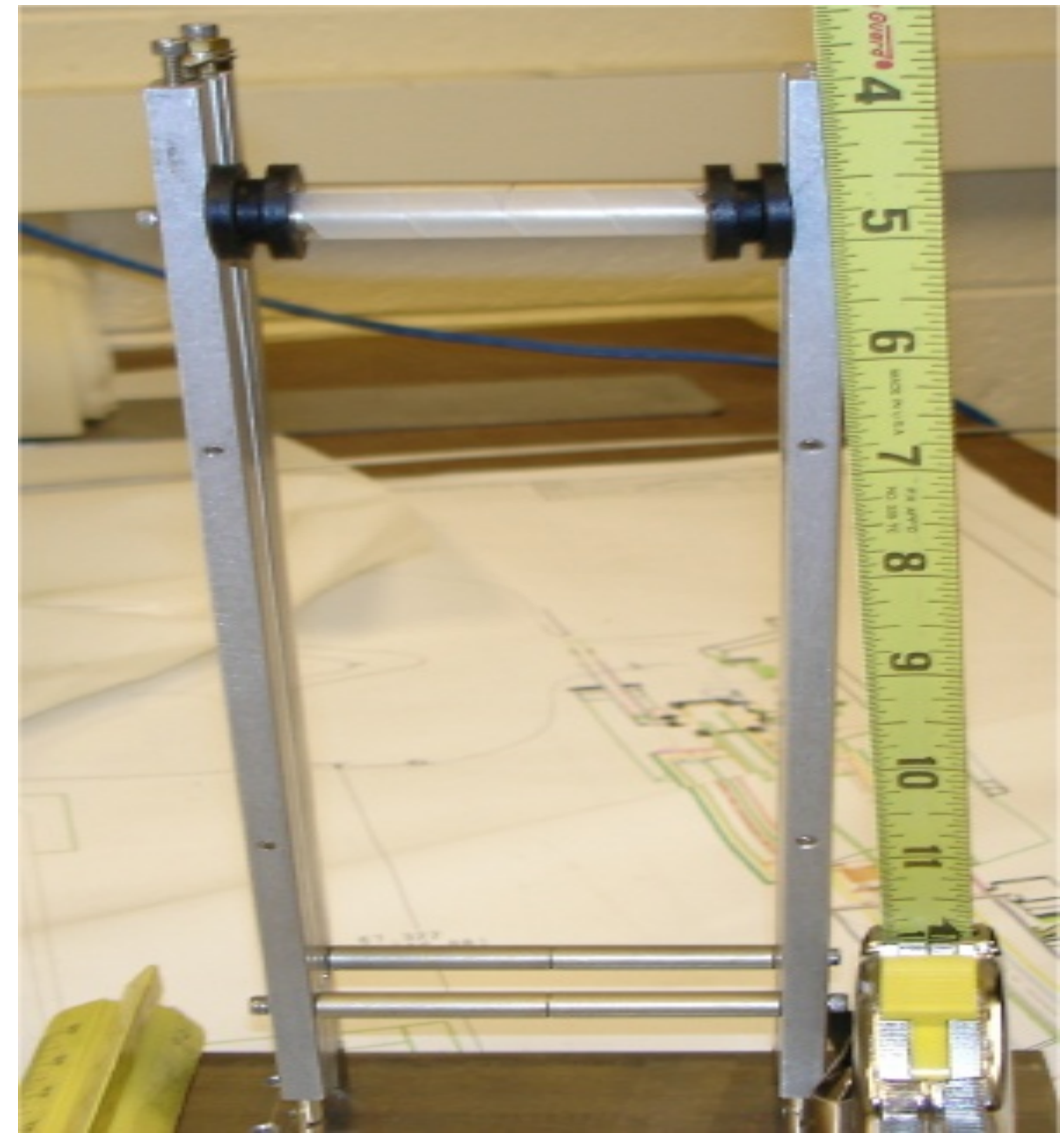

FIGURE 1. Irradiation sample holder.

The one uncertainty is the radiation resistance of the HTS material. There have been a few studies of the radiation tolerance of HTS materials [2,3], but they have been at fluxes lower than the estimated lifetime $10^{18} \mathrm{n} / \mathrm{cm}^{2}$ [4] that coils will be exposed to. The current material that is under consideration is BSCCO-2223, so we have undertaken irradiation of this material.

Because the only high neutron fluxes that are readily available are in reactors where the energy spectrum does not match the very high-energy neutrons that produce most of the dose in the magnet, we substitute protons from an accelerator. This will not give us a direct estimate of the expected lifetime, but the known sensitivity [5] of $\mathrm{Nb}_{3} \mathrm{Sn}$ to high-energy protons can be used as a reference.

\section{EXPERIMENT}

\section{Materials}

BSCCO-2223 tape from American Superconductor (ASC) and Sumitomo Electric Industries (SEI) were used for the irradiation studies. The tapes were about $4 \mathrm{~mm}$ wide and approximately $0.2 \mathrm{~mm}$ thick. The tapes were mainly silver (60-70\%) and did not have a stainless steel reinforcing. The stainless steel produces long-lived activities, so was not used. 


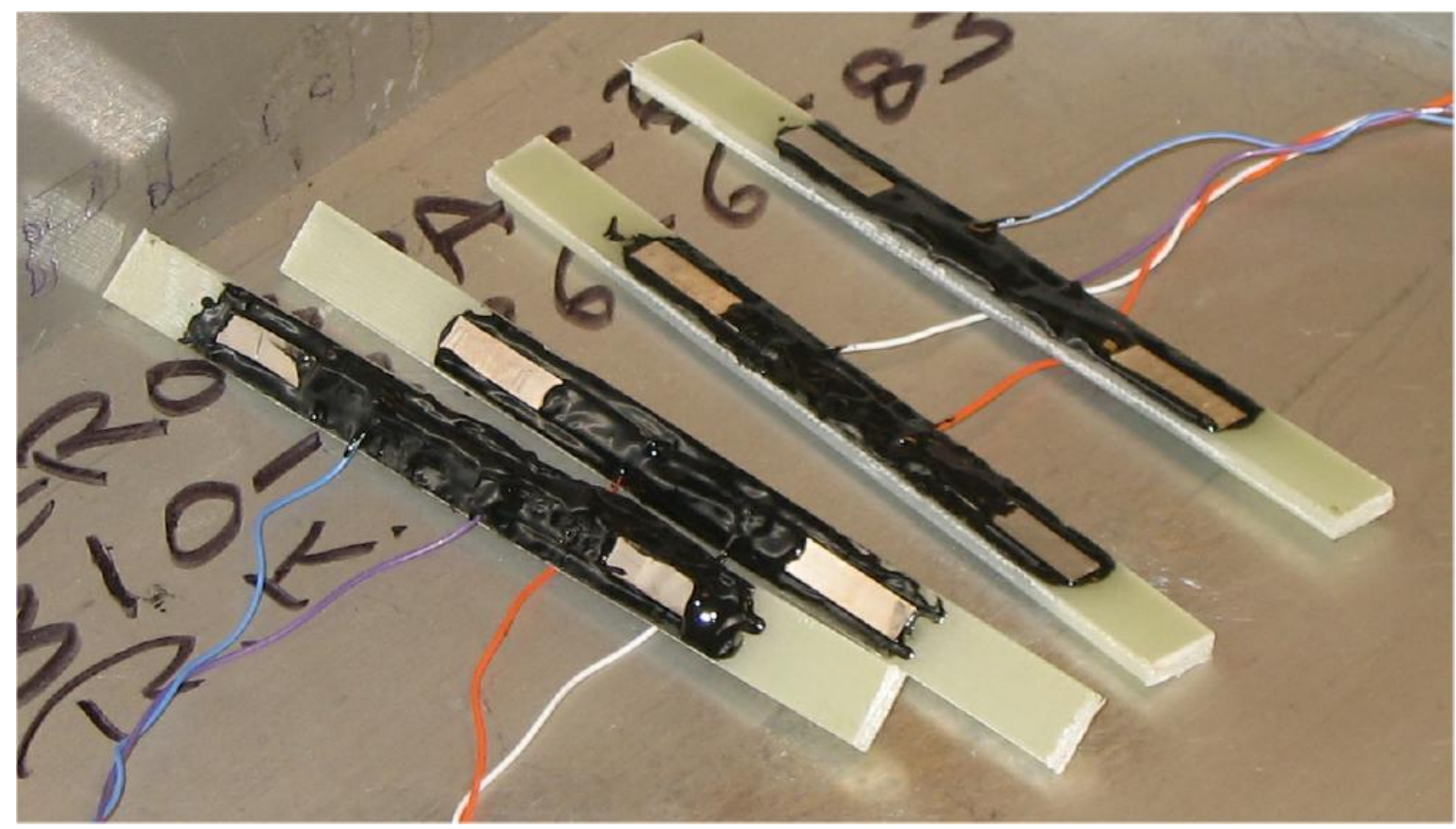

FIGURE 2. Samples after irradiation mounted on test supports. The samples are approximately 75 mm long.

\section{Irradiations}

Two samples of each tape were mounted on frames. The samples were approximately $75 \mathrm{~mm}$ long. Two sets of frames were used, one in front of the other, as shown in FIGURE 1. The rear frame could be dropped out of the beam by pulling a string that was run to a low-radiation area. Two samples were mounted next to each other, so the vertical beam size of $10 \mathrm{~mm}$ covered the combined $8 \mathrm{~mm}$ area. After approximately half of the irradiation, the rear sample was removed from the beam.

The irradiations were carried out at the LBNL's 88" Cyclotron using $50 \mathrm{MeV}$ protons. The average beam current was about $5 \mu \mathrm{A}$. The beam exited the beam line through a window into the air. After losing approximately $10 \mathrm{MeV}$ in the window, air and samples, the beam was stopped in a water-cooled stop. A fan was directed on the samples to keep them cool. The energy deposited in each sample was $10 \mathrm{~W}$. The total number of protons delivered was $2 \times 10^{18}$. After cooling for seven months, the samples could be handled safely.

\section{MEASUREMENTS}

\section{Radiographic}

After cooling, the samples together with one of each that had not been irradiated were mounted on G10 pieces with Stycast 2850 FT epoxy. The G10 holders have fibers in the plane of the tape so the thermal contraction of the BSCCO is matched, reducing the starin. Voltage taps were soft-soldered to the samples approximately $20 \mathrm{~mm}$ apart. Wire separation was measured for each sample to allow extraction of the $1 \mu \mathrm{V} / \mathrm{cm}$ criterion. Some of the samples are shown in FIGURE 2.

An autoradiogram of the samples was made to determine the irradiated area and each sample was analyzed using a Ge gamma detector and multichannel analyzer. A prominent silver-105 gamma line was used to determine the relative activation of each sample. The 


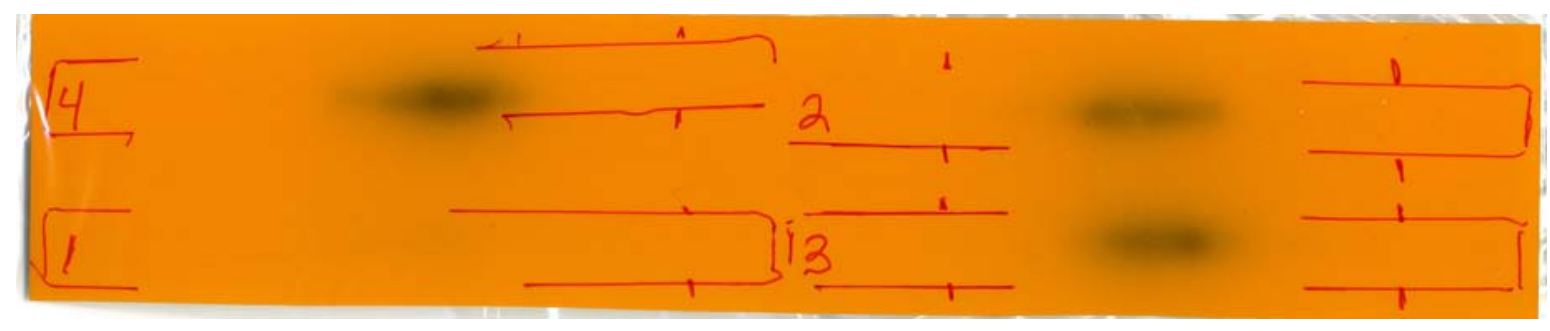

FIGURE 3. Radiogram of the samples made 7 months after irradiation. Dark spots on 2-4 show radioactivity.

TABLE 1. Activities of samples 7 months after irradiation in units of $\mu \mathrm{Ci}$ of ${ }^{107} \mathrm{Ag}$.

\begin{tabular}{cc}
\hline Sample & Activity $(\mu \mathrm{Ci})$ \\
\hline ASC-1 & 0.9 \\
SEI-1 & 11.7 \\
ASC-2 & 19. \\
SEI-2 & 20. \\
\hline
\end{tabular}

radiograms are shown in FIGURE 3. Samples are labeled according to the manufacturers.

Measurements of the size of the activated zone were used to determine the fluence (dose per unit area). TABLE 1 lists the measured activity of each sample in terms of the residual silver-105 activity.

\section{Critical currents}

Samples were tested in an open Dewar containing liquid nitrogen without an external magnetic field. Two examples are shown in FIGURE 4. The critical current was based on a criterion of $1 \mu \mathrm{V} / \mathrm{cm}$. Each sample was tested twice, but the variation in the short sample results differed by only $0.1 \mathrm{~A}$. The spacing of the voltage taps is a little uncertain because the soldered connections are not small with respect to the separation; i.e., the spacing could be $17 \pm 2 \mathrm{~mm}$. The effect on the critical currents is relatively small since the $n$-value is at least 5 .

\section{RESULTS}

All of the samples lost significant current transport $\left(\mathrm{I}_{\mathrm{c}}\right)$ as a result of the irradiations. The results given in TABLE 2 are given with respect to the assumed irradiation dose. The n-values are also given. It is obvious from the radiographic results shown in FIGURE 3 and in TABLE 1 that one sample, ASC-1, received very little fluence.

Before the samples were irradiated a filmstrip was briefly irradiated to assure the beam was correctly placed. Unfortunately, during the irradiation when only the two front samples were in place, a block supporting the frames broke. Because the area was very radioactive another film was not illuminated when the problem was fixed. It appears the positioning was not identical to the original one. This turned out to be fortuitous since

TABLE 2. Transport current in samples as a function of assumed dose, together with the n-values.

\begin{tabular}{ccc}
\hline Sample & $\mathbf{I}_{\mathbf{c}}$ (Amps) & n-value \\
\hline ASC reference & 66 & 9.5 \\
SEI reference & 82.7 & 10.3 \\
ASC-1 & 6. & 2.5 \\
SEI-1 & 15.1 & 5.3 \\
ASC-2 & 18.8 & 8.7 \\
SEI-2 & 25.9 & 6.3 \\
\hline
\end{tabular}


TABLE 3. Critical current ratios scaled with activity.

\begin{tabular}{cc}
\hline Sample & I/Iref \\
\hline ASC reference & 1 \\
SEI reference & 1 \\
ASC-1 & 0.1 \\
SEI-1 & 0.2 \\
ASC-2 & 0.3 \\
SEI-2 & 0.3 \\
\hline
\end{tabular}
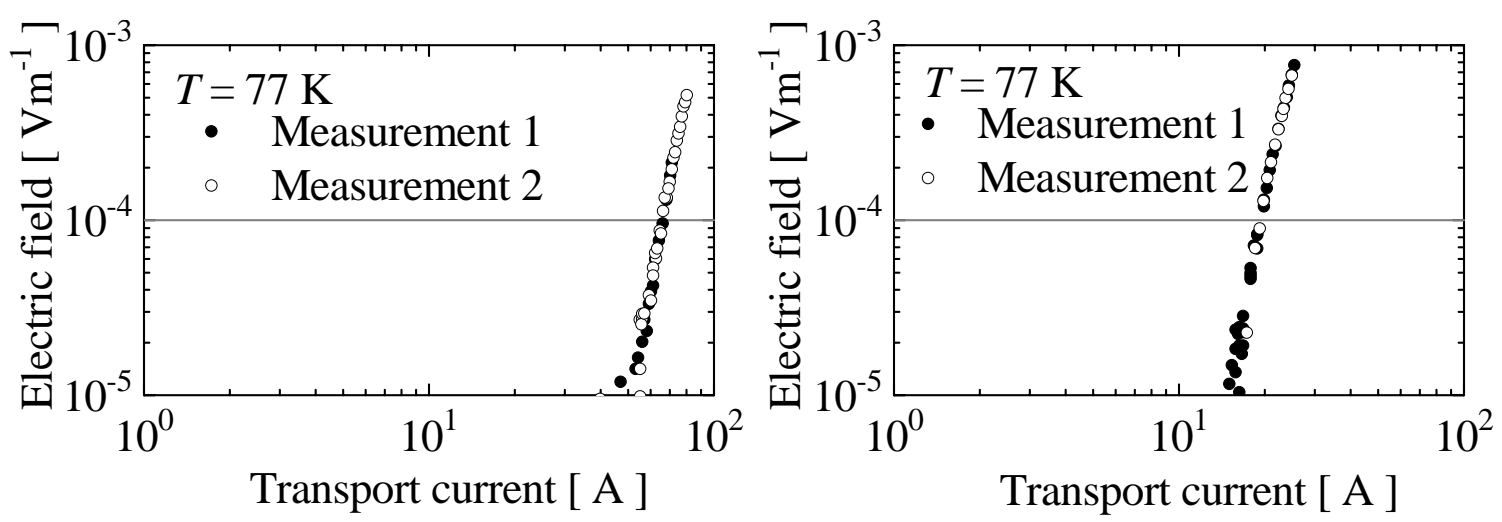

FIGURE 4. Voltage versus current measurements for the ASC reference sample and ASC-2.

ASC-1 got very little dose. The beam changed vertical position, so that SEI-1 received $3 \mathrm{x}$ $10^{17} \mathrm{p} / \mathrm{cm}^{2}$ before it was removed from the beam. The repositioned samples, ASC-2 and SEI-2, then received $5 \times 10^{17} \mathrm{p} / \mathrm{cm}^{2}$. This is consistent with the activities given in TABLE 1 . The results, scaled by the amount of 105-silver are given in TABLE 3 , together with the reference samples. They are presented as the ratio to the initial $I_{c}$ in the reference sample.

During the mounting of the samples, ASC-1 did not get securely attached to the G10 support. We believe the sample was not adequately supported during testing and the local strain caused a reduction in $\mathrm{I}_{\mathrm{c}}$. Given that it experienced little beam, the measurements on ASC-1 are not included in the final results

\section{COMPARISON WITH OTHER RESULTS}

Snead [5] measured the loss of $\mathrm{I}_{\mathrm{c}}$ in $\mathrm{Nb}_{3} \mathrm{Sn}$ using $30 \mathrm{GeV}$ protons. To compare with these results, we calculated the number of displacements per atom (dpa) by using the Monte Carlo Radiation Transport Code, PHITS [6,7]. The results are given in TABLE 4.

A previous study of high-energy neutron irradiation at $5 \times 10^{15} \mathrm{n} / \mathrm{cm}^{2}$ showed [2] no change in $I_{c}$. Reactor irradiation at fluence of $4 \times 10^{17} \mathrm{n} / \mathrm{cm}^{2}$ showed an enhanced $I_{c}$ [3]. A similar effect in $\mathrm{Nb}_{3} \mathrm{Sn}$ has been observed [8]. This enhanced $\mathrm{I}_{\mathrm{c}}$ is not observed in NbTi. No enhancement of $I_{C}$ was observed in our measurements, although the fluences were approximately the same as in [2].

\section{DISCUSSION}

Based on similar dpa's it appears that BSCCO-2223 is about a factor of two more sensitive to proton-induced damage than $\mathrm{Nb}_{3} \mathrm{Sn}$. There are several cautions that need to be considered with this statement. The irradiations were made at room temperature and might 
TABLE 4. Comparison of the results with those obtained after irradiation with $30 \mathrm{GeV}$-protons [5] on $\mathrm{Nb}_{3} \mathrm{Sn}$ at the same dpa. Data are the ratio $\mathrm{I} / \mathrm{I}_{0}$.

\begin{tabular}{ccc}
\hline dpa & $\mathbf{N b}_{3} \mathbf{S n}$ & BSCCO-2223 \\
\hline 0.0078 & 0.75 & 0.2 \\
0.013 & 0.5 & 0.3 \\
\hline
\end{tabular}

not apply to using the material at $20-30 \mathrm{~K}$. NbTi shows an annealing effect when it is first irradiated at $4 \mathrm{~K}$ and then warmed to room temperature before cooling and testing [5]. $\mathrm{Nb}_{3} \mathrm{Sn}$ does not show any annealing in the same cycle. We have assumed that BSCCO behaves like $\mathrm{Nb}_{3} \mathrm{Sn}$ and work by Ballarino [2] shows little difference between irradiation at room temperature and irradiation at $77 \mathrm{~K}$. However, it cannot be completely ruled out. If there is annealing, then the radiation resistance relative to $\mathrm{Nb}_{3} \mathrm{Sn}$ may be less than measured.

It is not completely clear that using displacement per atom is the correct way to obtain correlations between different irradiation systems. Thus while we can repeat the irradiation and simultaneously irradiate an $\mathrm{Nb}_{3} \mathrm{Sn}$ sample, we cannot make a good comparison with high-energy neutron irradiation. Snead [5] did compare NbTi irradiated with both $30 \mathrm{GeV}$ protons and reactor-spectra neutrons and observed very small differences. This gives some confidence the results are useful in predicting the lifetime of BSCCO-2223 in a high-energy neutron environment.

A more troubling issue is the low $\mathrm{I}_{\mathrm{C}}$ measured for samples that were not irradiated. The expected critical currents of both the ASC and SEI tapes is above $100 \mathrm{~A}$ at $77 \mathrm{~K}$. At present we do not have a good explanation for this discrepancy.

Since BSCCO-2223 is a first generation HTS material, it will shortly become unobtainable. Thus its use in a magnet that will be used a decade down the road is unlikely. New studies need to be carried out on materials like YBCO or BSCCO-2212. Studies made in 1991 [9] showed an increase in $\mathrm{J}_{\mathrm{c}}$ at reactor-spectra neutrons at fluences up to $2 \times 10^{18}$ $\mathrm{n} / \mathrm{cm}^{2}$ for YBCO. This is the appropriate fluence expected in the proposed fragment separators, although at a lower neutron-energy.

\section{SUMMARY}

Proton irradiations and subsequent critical current measurements on samples of BSCCO-2223 from two manufactures show a reduction in Ic that is about a factor of two lower than that observed in $\mathrm{Nb}_{3} \mathrm{Sn}$. In terms of using the material in a fragment separator, this is not a major problem, given the uncertainties in the measurements and interpretation. In the worst case the lifetime is reduced by a factor of two, meaning the magnets must be replaced every ten years instead of every twenty years. New studies on second-generation materials are needed before using HTS materials in any proposed fragment separator.

\section{ACKNOWLEDGEMENTS}

This work was supported in part by a grant from the US DOE, the State of Michigan and the NSF.

\section{REFERENCES}

1. Gupta, R., et al., IEEE Trans. on Applied Superconductivity 15, pp. 1148-1151 (2005).

2. Ballarino, A. et al., "Effect of fast neutron irradiation on current transport properties of HTS materials", http://at-mel-cf.web.cern.ch/at-mel-cf/resources/Irradiation_tests_Eucas.pdf. 
3. Hu, Q. Y., et al., Appl. Phys. Letters 65, pp. 3008-3010 (1994).

4. Zeller, A., Blideanu, V., Ronningen, R., Sherrill, B. and Gupta, R., "Radiation Resistant Magnets for the RIA Fragment Separator”, in Proc. Particle Accelerator Conference, ed. C. Horak, Published by IEEE, NJ, 2006, pp. 2200-2202.

5. Snead, C. L., Jr., J. Nuclear Materials 72, pp. 190-197 (1978).

6. Iwase, H., Kurosawa, T., Nakamura, T., Yoshizawa, N. and Funabiki, J., Nucl. Inst. Meth Phys. Res. B183, pp. 374-382 (2001).

7. Iwase, H. Ph. D Thesis, Tohuku University, Japan, 2003.

8. Weiss, F., Fluekinger, R., Maurer, W., Hahn, P. A. and Guinan, M. W., IEEE Trans. on Mag. MAG-23, pp. 976-979 (1987).

9. Lessure, H. S., et al., J. Appl. Phys. 70, pp. 6513-6515 (1991). 